\title{
Implementation of remotely controllable Mobile Robot System over Mobile Ad-hoc Network
}

\author{
Mohammad Shahidul Hasan \\ Faculty of Computing, Engineering and Sciences \\ Staffordshire University, UK \\ m.s.hasan@staffs.ac.uk
}

\begin{abstract}
Remotely controllable wireless mobile robots are being used in many applications now days. Such systems can be restricted by the range of wireless signal. Mobile Ad-hoc Networks offer a number of advantages e.g. multi hop communication i.e. longer range; can be deployed without any infrastructure etc. This paper presents the implementation details of a wireless mobile robot system over Mobile Ad-hoc Network. It consists of a target mobile robot and a relay / observer robot. The target robot is equipped with a robotic arm to execute various tasks. The relay / observer robot can route network packets between the controller and the target robot. It also produces visual feedback of the target robot to the user at the controlling end.
\end{abstract}

Keywords: Wireless Mobile Robot, Mobile Ad-hoc Network, tele-operation, Autonomous movement etc.

\section{INTRODUCTION}

Today, robots are being applied in many fields of service; ranging from housekeeping to space exploration. They are expected to work with humans by sharing various service tasks [1]. Such systems are also suitable for military use, rescue operations, and exploring hazardous environment [2] e.g. nuclear decommissioning, executing tele-surgery etc. Normally mobile robots operate in tele-operated or autonomous mode. In the tele-operated mode, a user can observe the camera output, watch sensor data, and guide the robot manually using a device e.g. joystick attached to the controlling computer. In the autonomous mode, the robot does not receive any command from the user and an intelligent control algorithm can navigate it autonomously based on the sensor data. The difficulties of autonomous navigation are caused by the complexity and uncertainty of the environment model as well as the inconsistency between an inaccurate robot model and control in real-time [2]. In the presence of many scattered obstacles or in critical applications where intelligent decisions are required dynamically, the autonomous mode cannot be relied on. Instead a tele-operated control system where a human user directly controls the robot according to his decision is utilised [3].

Heavy engineering mobile robots that are controlled using wired communication restrict the coverage area and offer very limited flexibility for many applications. Therefore, teleoperated mobile robots must allow the control using wireless communication to provide the freedom from wires and to

\author{
Hongnian $\mathrm{Yu}$ \\ Faculty of Science and Technology \\ Bournemouth University, UK \\ yuh@bournemouth.ac.uk
}

support higher degree of mobility. With the development of high speed-reliable portable computing devices and wireless networks, control applications can be now implemented the using the existing low-cost e.g. IEEE 802.11, low-power e.g. Bluetooth etc. technologies. The IEEE 802.11 standard also supports multi-hop ad-hoc configuration known as Mobile Adhoc Networks (MANET) which is shown in Figure 1. Each node in an ad-hoc network is equipped with a transceiver, an antenna and a power source [4]. MANETs are self-organising and can be easily deployed without any infrastructure [5]. Nodes perform routing operations and are capable of maintaining the dynamic topology of the network. Routes can have multiple hops between the source and the destination node; therefore, a MANET provides a suitable dynamic framework for distributed applications. Furthermore, it can reconfigure after a failure and offers a high degree of flexibility. Ad-hoc networks have been widely used in sensor networks where devices are equipped with small, low cost and low power transceivers and sensors [4], [6]. Such systems are useful in both home and industrial environments and can be implemented using the existing devices e.g. desktop computers, laptops, PDAs or other devices that support the IEEE 802.11.

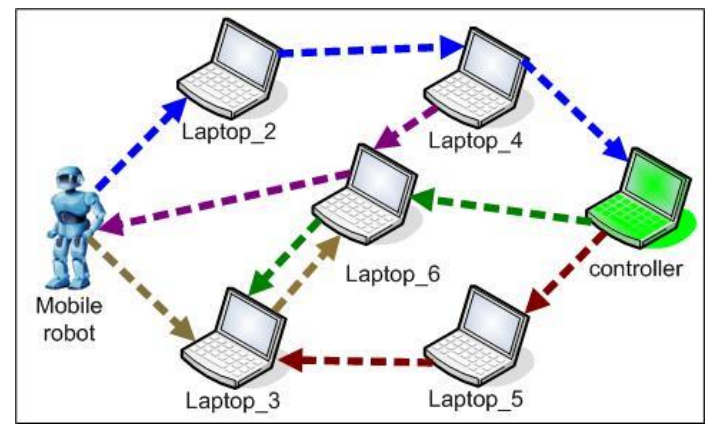

Figure 1: A tele-operated mobile robot over a MANET.

Nowadays, tele-operated mobile robots can support cameras to provide visual feedback at the user end. However, cameras only mounted to the operating mobile robot itself might provide limited or restricted views. To have a clear understanding of the operating environment, it is necessary to have side, front views etc. of the operating mobile robot from cameras attached to other mobile robots to execute critical tasks precisely. 
The aim of this research is to design and implement a teleoperated mobile robot system over MANET based on the IEEE 802.11 standards for industrial applications with distance and multiple video feedback support. The multiplehop feature of MANET also extends the operating range of the system at the cost of longer network delay. The authors presented a demonstration in an exhibition hosted by a UK based nuclear decommissioning company, where other universities and companies in robotics made presentations. The demonstration system made by the Staffordshire University team involved a mobile robot and an observer robot being controlled over a MANET. This paper presents the design of the system.

\section{PREVIOUS RESEARCH}

Paper [2] presents a three-level architecture for a vehicle based intelligent mobile robot that can demonstrate road following, road obstacle avoidance etc. The architecture and the typical functions are shown in Figure 2. The performance of a robot's trajectory following tasks is investigated in [7] where the wireless mobile robot and network are simulated by Simulink and Opnet, respectively. The paper uses a 2-level controller to follow the desired trajectories.

Level 3: Intelligent level or planning system Receiving mission command, building task and environment model, path planning etc.

\begin{tabular}{|} 
Level 2: Coordinate level or monitor system \\
Road following and obstacle avoidance, human machine \\
interface etc. \\
Level 1: Executive level \\
$\begin{array}{c}\text { Sonar range finder system, position system, optical encoders, } \\
\text { vehicle control command executing etc }\end{array}$ \\
\hline
\end{tabular}

Figure 2: The architecture and functions of an intelligent mobile robot [2].

A restaurant service mobile robot equipped with camera, speaker, sensors and laser positioning system for fast and precise positioning and guidance is developed in [1] to transfer dishes to the customers. In paper [3], a bidirectional teleoperation system is presented where the slave robot receives the user's command through the joystick and sends back the environment information to the user as a feedback force. A full-duplex point-to-point communication between a PC and the mobile robot using Bluetooth technology is used to implement the real time operation in [8]. However, it has the limitation of short range of Bluetooth which is approximately $10 \mathrm{~m}$. A Bluetooth piconet based mobile robot system is proposed in [9] where the master or host mobile robot can connect to four slave mobile robots in the ad-hoc mode. The master mobile robot can also connect to an access point to communicate with a wired network e.g. a LAN. One downside of the system is that up to eight devices can be active in a piconet and only one device could be the master. A mobile robot with four LCD panels, eight cameras, four microphones, forty ultrasonic sensors and two arms is developed in [10] using off-the-shelf components for advanced video conferencing to reduce business travel. However, the system requires high wireless communication bandwidth to support multiple audio and video streams along with the control commands. Paper [11] develops two non-linear controllers for the DC motors of a robot where a DSP controls the servo motors and a PDA implements the algorithms with complex computations. A tele-operated system based on HTTP through the Internet is developed in [12] for a robotic arm equipped with camera. Multiple servers are used to authenticate users, to translate user mouse clicks in 2-D workspace into robot commands and to execute robot actions. A TCP and web browser based tele-operated mobile robot system has been developed in [13] using Virtual Reality Modelling Language (VRML) which can transmit 3-D models of objects. The users can use the tele-operation system from any Internet site without any specialised software and can support applications such as tele-maintenance of home, tele-gardening, telesignature etc. The mobile robot presented in [14] has laser scanner, sonar, a camera and considered two types of force feedback: environmental and collision-preventing. The onboard controller performs close-range obstacle avoidance while the user uses the haptic feedback device to dictate the desired navigation. The performance of the system is investigated in virtual environment as well as in the real world.

\section{PRELIMINARIES}

This section presents the specifications of the components that have been used in the implementation.

\section{A. The DRK8080 robot}

The DRK8080 which is shown in Figure 3 is an integrated robotic system that supports wireless communication. Various data e.g. image, audio, sensor information etc. are available to the user through a set of ActiveX control components (SDK) developed for Microsoft Visual $\mathrm{C}++$ and Visual Basic programming environment. A user can also send various control commands to the robot. Multiple applications are allowed to access the sensor data simultaneously. It supports different types of sensors e.g. infrared, temperature, acceleration and tilting, five servo motors for head, neck and mouth movement, LCD, camera, speaker and microphone [15].

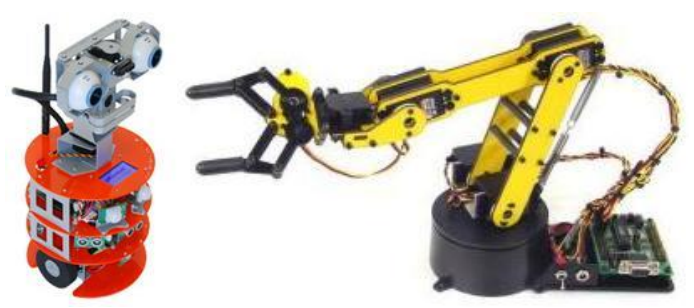

Figure 3: The DRK8080-compact WiFi Mobile robot [15] and the Lynx 6 robotic arm [16].

\section{B. The Lynx robotic arm}

The Lynx robotic arm, shown in Figure 3, delivers fast, accurate, and repeatable movements. The robot features; base rotation, shoulder, elbow, wrist motion, wrist rotate, and a functional gripper. Therefore, it supports five Degrees Of 
Freedom (DOF) with wrist rotate. The entire robot arm uses seven servo motors; one for the base, two for the shoulder, one for the elbow, one for the wrist and two for the gripper [16]. Servos 0 to 5 allow base rotation, shoulder up-down, elbow up-down, wrist up-down, gripper rotation and gripper openclose movements.

\section{The target mobile robot}

Most mobile robots are designed as embedded systems without any operating system. To support flexible architecture by allowing execution of complex and large algorithms, incorporation of image, video and voice handling capability, a $\mathrm{PC}$ is necessary as the heart of the robot [9]. Therefore, a PC based custom built mobile robot has been developed which is used as the target robot and is shown in Figure 4. The mobile robot has four wheels and three decks. The bottom deck contains a $12 \mathrm{~V}$ lead acid battery, two DC motors to drive each wheel via a gearbox, fuses from the battery to the embedded controller, and 500ppr encoders attached directly to each wheel shaft. Differential steering is accomplished by driving each wheel separately allowing turning on the spot, curves and forward/backward motion of the robot like [1], [3], [8] etc.

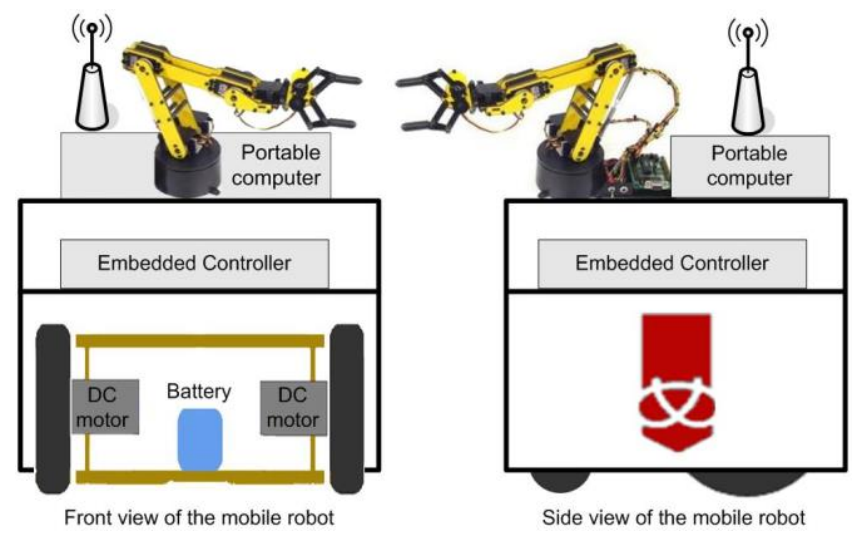

Figure 4: The custom built mobile robot with robotic arm and a portable computer.

The middle deck contains an embedded microcontroller, three ultrasonic transducers, led indicators, and a motor driver for the DC motors. The microcontroller is a PIC18F4550 [17] which is connected to the PC through the USB interface. The microcontroller runs a program with a PID algorithm that rotates the DC motors at a desired rate. This rate is the encoder pulses attached to the motors at a sample rate of $100 \mathrm{~ms}$. The motor driver is capable of driving the motors using PWM up to $5 \mathrm{~A}$ and is type MD22 available from Robot Electronics [18]. The data available to the PC is: the desired motor rate, the actual motor rate, the distance reported by the sonic sensors, and the battery voltage. The PC can send the desired motor rates to the microcontroller in the range of $0-100$. The mechanism of closed loop feedback control to continuously adjust the motor torque is found in [11], [19], [20] etc. The two front passive or caster wheels which can rotate freely to follow the differential steering are used to balance the robot. The differential steering with caster wheel configuration allows the robot to turn in place which human being is capable of doing [10]. The ultrasonic transducers provide distance feedback for collision avoidance as only camera based teleoperated mobile robots can suffer from limitations such as shadowed and curved areas are not viewable properly, bad weather effects, the camera view etc. [3].

To add gripping capability to the mobile robot, the Lynx 6 robotic arm has been installed at the front side on the top deck. A light weight portable computer based on Mini-ITX mainboard [21] and equipped with Belkin Wireless network card is also attached to the top deck. The robotic arm is connected to the COM port of the portable computer to receive commands. The mobile robot is interfaced to the on-board portable computer through USB connection with a serial port emulator. It is detected by the computer as COM port when connected. The battery is capable of powering the mobile robot, the robotic arm and the portable computer.

The commands that the mobile robot takes from the computer are shown in Table I. Each command string has the format $l \mid r$.Speed where $l$ or $r$ denotes the left or right wheel motor and Speed is the rotation speed of the motor. Positive and negative values for the speed will apply forward and backward rotation, respectively. For example, two left and right wheel forward commands, i.e. 120 and $r 20$ will move the robot forward at the speed of 20 pulses per interrupt. Similarly, $l-10$ and $r-10$ will move the robot backward. As the robot supports differential steering, the commands $l 30$ and $r$ 30 will make the robot to turn right. To turn left, $l-30$ and $r 30$ can be sent to the robot.

Table I: The commands for the custom built mobile robot.

\begin{tabular}{|l|l|l|l|}
\hline Command & Range & Format & Example \\
\hline $\begin{array}{l}\text { Rotate left } \\
\text { wheel }\end{array}$ & $\begin{array}{l}\text { Forward } \\
(0 \text { to 100) } \\
\text { Reverse } \\
(-100 \text { to 0) }\end{array}$ & lspeed & 120,15 etc. \\
\hline $\begin{array}{l}\text { Rotate right } \\
\text { wheel }\end{array}$ & $\begin{array}{l}\text { Forward } \\
(0 \text { to 100) } \\
\text { Reverse } \\
(-100 \text { to 0) }\end{array}$ & rspeed & r30, r5 etc. \\
\hline
\end{tabular}

\section{End-to-end connection}

Computer network communication relies on the Internet protocol (IP) architecture which is based on a connectionless end-to-end datagram service using IP packets. Above the IP protocol, two transport layer protocols; Transmission Control Protocol (TCP) and User Datagram Protocol (UDP) are available for different applications [22]. The protocol stack is shown in Figure 5. 


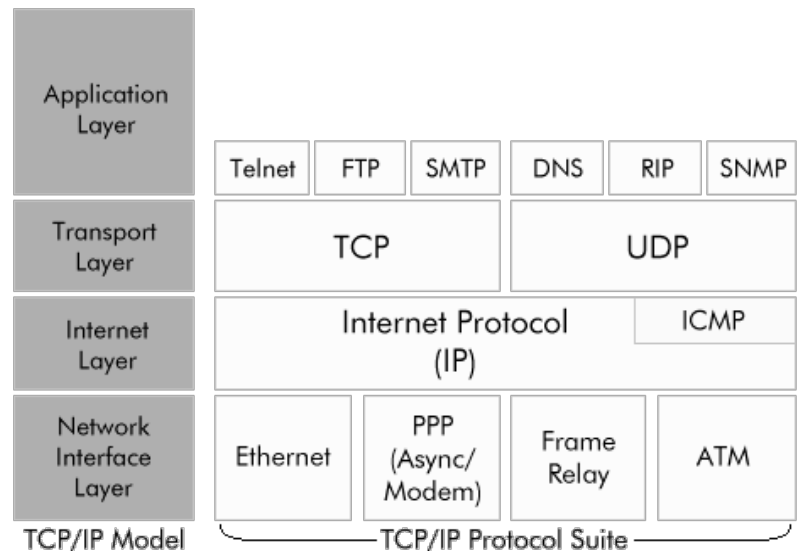

Figure 5: The TCP/IP model [23].

The TCP is not appropriate for MANET as it uses connection oriented packet transfer [24]. The TCP based protocols e.g. HTTP offers no transmission time guarantees and hence it is NOT suitable for time-critical interactions [12]. For real-time applications, UDP is shown more suitable than the TCP in terms of delay and delay jitter [22] as shown in Figure 6. Furthermore, the retransmission mechanism of the TCP will simply produce unwanted network traffic as the control application carries real time data. On the other hand, UDP offers low overheads as it does not maintain connections and discards obsolete packets instead of retransmissions. Therefore, it is preferable for networked control applications [25].

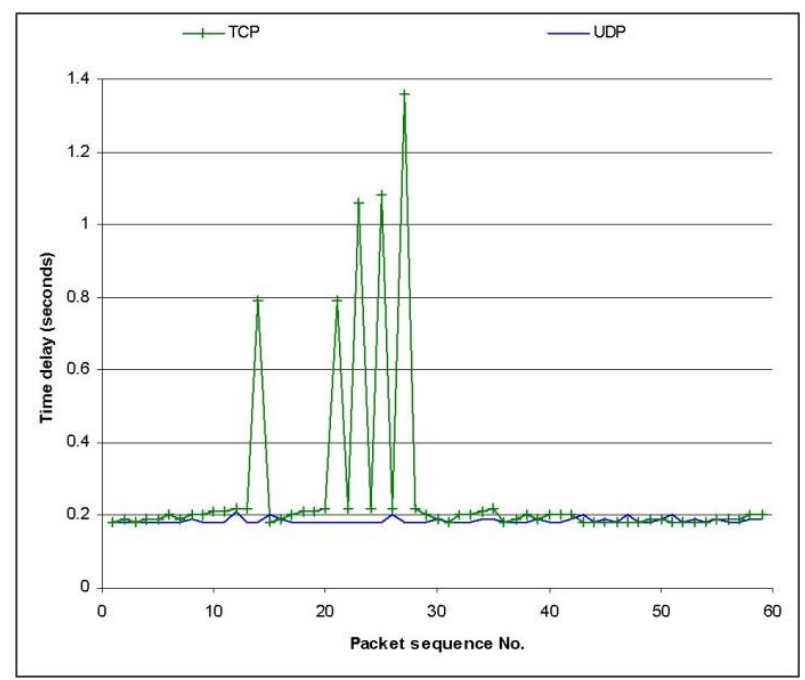

Figure 6: The comparison of delay and jitter between the TCP and UDP [22], [26].

\section{IMPLEMENTATION DETAILS}

The overall design of the system is shown in Figure 7. The laptop runs the control applications for both the target and the DRK8080 mobile robot. As the DRK8080 does not have the ability of packet routing, a WiFi enabled handheld computer has been attached to it so that it can offer the capability of a standard mobile network node. The wireless modules of the laptop, the handheld PC and the computer on the mobile robot are configured to communicate in ad-hoc mode. The network packets are transmitted from the controller laptop to the handheld PC and routed to the target mobile robot.

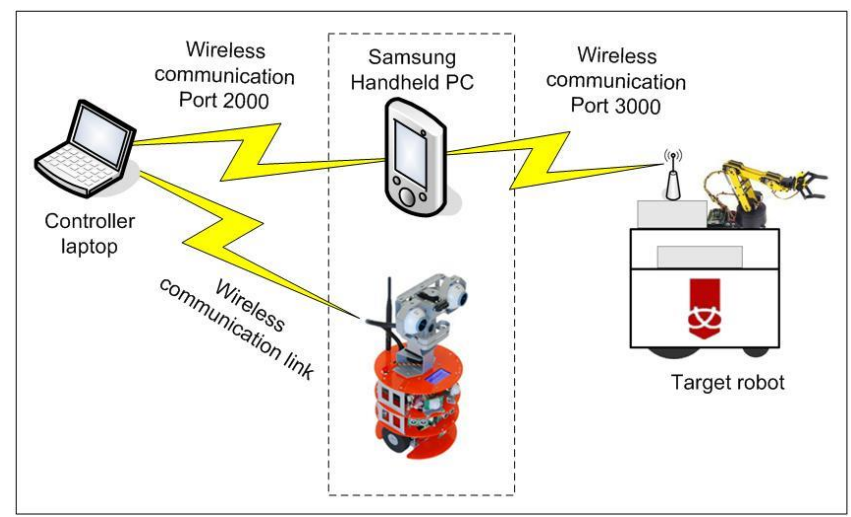

Figure 7: The overall system design for the tele-operated mobile robot.

The DRK8080 mobile robot is equipped with a camera and six ultrasonic sensors. The camera is used to provide visual feedback of the target robot at the controller end. The DRK8080 can be moved to various positions to observe or monitor the target robot from different angles. The sonar sensors can be used to sense the distances from surrounding objects to avoid collisions or to make the DRK8080 autonomous. Hence, the DRK8080 provides two functionalities:

- $\quad$ Routing packets between the controller and the target robot to have extended range.

- Monitor the activity of the target robot from the controller end using the camera.

\section{A. Network communication mechanism}

The controller-to-handheld computer and handheld computer-to-target robot communications have been implemented using $C$ socket and the UDP as shown in Figure 7. Such socket communication between the remote and local sites is also implemented in [13]. The handheld computer runs an application to receive commands from the controller using port 2000. As soon as it gets a command from the controller it routes the command to the target robot mobile robot computer. The portable computer runs another application that listens to the port number 3000 to receive commands from the handheld computer. To avoid blocking for receiving socket applications, select function has been utilised to specify a timeout of five seconds. The port numbers 2000 and 3000 for the communications are chosen randomly.

\section{B. Network Packet format}

A network packet format containing two fields, shown in Figure 8, has been used to support both controller-to-handheld computer and handheld computer-to-target robot communications. The format supports commands for the mobile robot as well as the robotic arm. The description of the packet format and the allowed values are given in Table II. For example, to rotate the base of the robotic arm (servo 0) to right, the value of 0 (servo 0 ) and $1(\mathrm{Up})$ are put into field 1 
and field 2, respectively. Again, the values of 100 and 3 in the fields of Robotic arm/Mobile Robot and Command, respectively will make the mobile robot go backward.

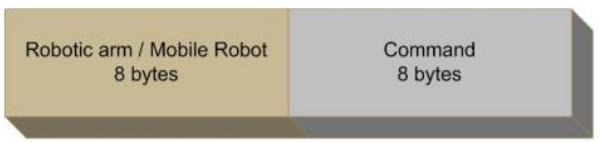

Figure 8: The packet format for socket communication.

Table II: Socket communication packet format and allowed values

\begin{tabular}{|c|c|c|}
\hline Device & $\begin{array}{l}\text { Field 1: } \\
\text { Mobile robot or } \\
\text { robotic arm }\end{array}$ & $\begin{array}{l}\text { Field 2: } \\
\text { Command }\end{array}$ \\
\hline $\begin{array}{l}\text { Robotic arm } \\
\text { (servo } 0 \text { to 5) }\end{array}$ & Value: 0 to 5 & $\begin{array}{l}1=\mathrm{Up} \\
2=\text { Down }\end{array}$ \\
\hline Mobile robot & Value: 100 & $\begin{array}{l}1=\text { Forward } \\
2=\text { Right } \\
3=\text { Backward } \\
4=\text { Left } \\
5=\text { Stop }\end{array}$ \\
\hline
\end{tabular}

C. The user interface for the tele-operated mobile robot control

The user interface to control both the target robot and the DRK8080 is shown in Figure 9. The DRK8080 movement and DRK8080 head movement are used to provide different viewing positions and angles of the target robot.

- Target robot viewing window: the target robot can be monitored using the camera mounted on the DRK8080 through the viewing window of the user interface. The camera supports a resolution of $352 \times$ 288 [15].

- Target robot movement control: forward, backward, right, left and strop command buttons.

- Target robot arm control: arm base left-right, shoulder up-down, elbow up-down, wrist up-down, gripper rotation and gripper open-close command buttons.

- The DRK 8080 relay/observer robot sensor readings: the readings of six distance sensors (front left, front, front right, back left, back, back right) on the DRK8080 are available on the user interface to avoid collision.

- The DRK 8080 relay/observer robot head movement control: head up-down, left-right and default position command buttons.

- The DRK 8080 relay/observer robot movement control: Forward, backward, right, left and strop command buttons.

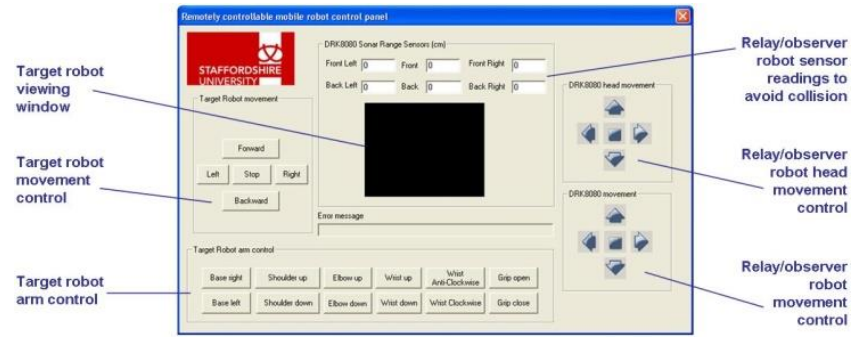

Figure 9: The user interface for the tele-operated mobile robot control.

\section{FUTURE WORK}

The following ideas can be proposed as direction of future work.

- Replacing the buttons of the user interface by a joystick to improve the controllability.

- Introducing the haptic feedback mechanism to improve user's performance in terms of number of collisions, navigation time etc. as discussed in [3], [14].

- An easy way to support the video capability on the target robot is by installing an instant messaging application e.g. MSN messenger and a web camera on the portable computer.

- Allowing interactive movement. When the user clicks at a particular point on the camera window, the robot will move to the corresponding point. It can be implemented by treating the camera window as the rectangular user interface that is presented in [12].

- Autonomous navigation of the mobile robot from point A to point B using on-board ultrasonic sensors and closed loop control.

- Autonomous tracking of the mobile robot by the observer robot i.e. the DRK8080 using image extraction and object recognition technique.

- As radio signals can be received by all nearby receivers, unauthorised users can exploit the resources of the tele-operated system. Strong encryption and authentication mechanisms are required for the security of the system.

- $\quad$ The entire system of Figure 7 can be expanded as shown in Figure 10 to accommodate multiple target robots. Some DRK8080 robots play the role of routing nodes and some play the role of observer as well as router.

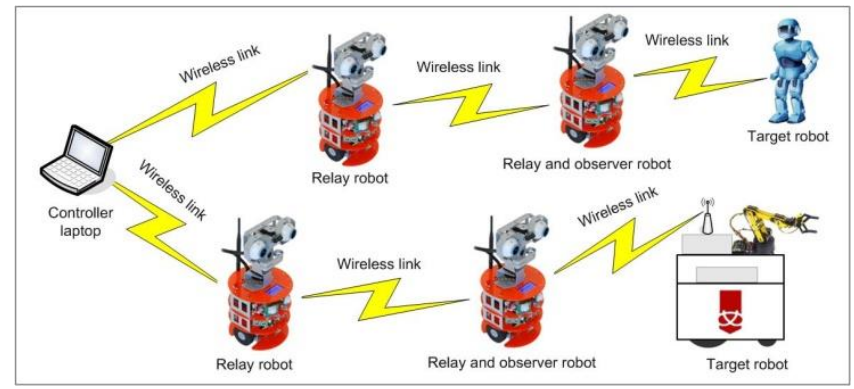

Figure 10: The extended tele-operated mobile robot system with multiple target robots. 


\section{CONCLUSION}

The paper presents the implementation details of a wireless mobile robot system over a MANET using video feedback to the user at the controller end. Both the target and the relay/observer robots and the controller can be mobile in this design. The intermediate DRK8080 robot routes packets to the target robot as well as providing visual feedback to the controller laptop.

\section{REFERENCES}

[1] J. H. Tzou and K. L. Su, "The Development of the Restaurant Service Mobile Robot with a Laser Positioning System," presented at the 27th Chinese Control Conference, Kunming, China, 2008, pp. 662-666.

[2] K. He, H. Sun, M. Guo, and H. Wang, "Research of intelligent mobile robot key techniques," presented at the IEEE International Conference on Industrial Technology, Shanghai, China, 1996, pp. 503-507.

[3] S. Han and J. Lee, "Tele-operation of a Mobile Robot Using a Force Reflection Joystick with a Single Hall Sensor," presented at the 16th IEEE International Symposium on Robot and Human interactive Communication, Korea, 2007, pp. 206-211.

[4] S. Kumar, V. S. Raghavan, and J. Deng, "Medium Access Control protocols for ad hoc wireless networks: A survey," Ad Hoc Networks (Elsevier) Journal, vol. 4, no. 3, pp. 326-358, 2006.

[5] E. M. Royer and C. K. Toh, "A Review of Current Routing Protocols for Ad Hoc Mobile Wireless Networks," IEEE Personal Communications, vol. 6, no. 2, pp. 46-55, 1999.

[6] F. D. Pellegrini, D. Miorandi, S. Vitturi, and A. Zanella, "On the Use of Wireless Networks at Low Level of Factory Automation Systems," IEEE Transactions on Industrial Informatics, vol. 2, no. 2, pp. 129143, 2006.

[7] Y. Liu, M. S. Hasan, and H. Yu, "Modelling and Remote Control of an Excavator," International Journal of Automation and Computing (IJAC), vol. 7, no. 3, pp. 349-358, Aug. 2010.

[8] S. H. Choo, S. H. M. Amin, N. Fisal, C. F. Yeong, and J. Abu Bakar, "Using Bluetooth transceivers in mobile robot," presented at the Student Conference on Research and Development, Malaysia, 2002, pp. $472-476$.

[9] S. H. Choo, S. H. M. Amin, N. Fisal, C. F. Yeong, and J. Abu Bakar, "Development of PAN (personal area network) for mobile robot using Bluetooth transceiver," presented at the Student Conference on Research and Development, Malaysia, 2003, pp. 160-165.

[10] N. P. Jouppi, "First steps towards mutually-immersive mobile telepresence," presented at the 2002 ACM conference on Computer supported cooperative work, New Orleans, USA, 2002, pp. 354-363.

[11] C. F. Chang, C. I. Huang, and L. C. Fu, "Nonlinear control of a wheeled mobile robot with nonholonomic constraints," presented at the IEEE International Conference on Systems, Man and Cybernetics, Hague, Netherlands, 2004, vol. 6, pp. 5404-5410.
[12] K. Goldberg, S. Gentner, C. Sutter, and J. Wiegley, "The Mercury Project: A Feasibility Study for Internet Robots," IEEE Robotics and Automation Magazine, vol. 7, no. 1, pp. 35-40, 2000.

[13] H. Hirukawa, T. Matsui, H. Onda, K. Takase, Y. Ishiwata, and K Konaka, "Prototypes of Teleoperation Systems via a Standard Protocol with a Standard Human Interface," presented at the IEEE Intemational Conference on Robotics and Automation, USA, 1997 vol. 2, pp. 1028-1033.

[14] S. Lee, G. Sukhatme, G. J. Kim, and C. M. Park, "Haptic teleoperation of a mobile robot: a user study," Presence: Teleoperators and Virtual Environments, vol. 14, no. 3, pp. 345-365, 2005.

[15] "WiRobot DRK6080/8080 User Manual," 2006. [Online]. Available: http://www.drrobot.com/products/item_downloads/DRK8080_1.pdf. [Accessed: 08-Feb-2009].

[16] "Lynx 6 Series of Robotic Arms," 2009. [Online]. Available: http://www.lynxmotion.com/Category.aspx?CategoryID=25. [Accessed: 08-Feb-2009].

[17] "PIC18F2455/2550/4455/4550 Data Sheet," 2007. [Online]. Available:

http://ww1.microchip.com/downloads/en/DeviceDoc/39632D.pdf. [Accessed: 13-Aug-2009].

[18] “MD22 - Dual 24Volt 5Amp H Bridge Motor Drive," 2009. [Online]. Available: http://www.robot-electronics.co.uk/htm/md22tech.htm. [Accessed: 13-Aug-2009].

[19] J. Velagic, B. Lacevic, and N. Osmic, "Nonlinear Motion Control of Mobile Robot Dynamic Model," in Mobile Robots Motion Planning, New Challenges, Vienna: Advanced Robotic System International, 2008, pp. 531-552.

[20] B. Lacevic, J. Velagic, and B. Perunicic, "Reduction of Control Torques of Mobile Robot Using Hybrid Nonlinear Position Controller," presented at the International Conference on Computer as a Tool, EUROCON 2005, Serbia \& Montenegro, Belgrade, 2005, pp. 314-317.

[21] "VIA Mini-ITX Mainboard Form Factor: 17cm x 17cm," 2009. [Online]. Available: http://www.via.com.tw/en/initiatives/spearhead/mini-itx/. [Accessed: 13-Aug-2009].

[22] P. X. Liu, M. Meng, X. Ye, and J. Gu, “An UDP-Based Protocol for Internet Robots," presented at the 4th World Congress on Intelligent Control and Automation, China, 2002, vol. 1, pp. 59-65.

[23] A. S. Tanenbaum and D. J. Wetherall, Computer Networks, 5th ed. New Jersey: Prentice Hall, 2011.

[24] M. Conti and S. Giordano, "Multihop Ad Hoc Networking: The Reality," IEEE Communications Magazine, vol. 45, no. 4, pp. 88-95, 2007.

[25] N. J. Ploplys, P. A. Kawka, and A. G. Alleyne, "Closed-loop Control over Wireless Networks," IEEE Control Systems Magazine, vol. 24 no. 3, pp. 58-71, 2004.

[26] P. X. Liu, M. Q. H. Meng, P. R. Liu, and S. X. Yang, "An End-to-End Transmission Architecture for the Remote Control of Robots Over IP Networks," IEEE/ASME Transactions on Mechatronics, vol. 10, no. 5, pp. 560-570, 2005. 